\title{
Renormalization Group and Analyticity in one Dimension: A Proof of Dobrushin's Theorem
}

\author{
M. Cassandro ${ }^{1}$ and E. Olivieri ${ }^{2}$ \\ 1 Istituto di Fisica, Università di Roma, CNR (G.N.S.M.), I-00100 Roma, Italy \\ 2 Istituto di Matematica, Università di Roma, CNR (G.N.F.M.), I-00100 Roma, Italy
}

\begin{abstract}
We consider one dimensional systems described by many body potentials with finite first moment and prove that the correlation functions are analytic in the interaction parameters. This result is not new (Dobrushin, 1973) but our proof is simpler and physically more transparent. We show that by introducing suitable blocks and averaging over the variables associated to a subset of the blocks (decimation procedure), the resulting effective interaction is such that the system can always be dealt with as a high temperature system.
\end{abstract}

\section{Introduction}

In this paper we will deal explicitly with the lattice gas case only; the extension to other cases is considered in Sect. 3.

Consider the elements of $\mathbb{Z}$ as the sites of a one-dimensional lattice, each site may be occupied by 0 or 1 particle. Call $K_{A}$ the product of one copy of the set $\{0,1\}$ for each point of the set $\Lambda \subset \mathbb{Z}$ and $K=\{0,1\}^{\mathbb{Z}} \cdot x_{\Lambda} \in K_{\Lambda}$ is a configuration of occupied and empty sites in $\Lambda$.

The energy of a finite volume $\Lambda$, when $\mathbb{Z} \backslash \Lambda$ is empty, is given by:

$$
H_{\Lambda}^{\Phi}\left(x_{\Lambda}\right)=\sum_{X \subset A} \Phi(X) \prod_{t \in X} x_{t},
$$

where the potential $\Phi$ is a real or complex function defined on the finite subsets of $\mathbb{Z}$. In the sequel we will consider potentials belonging to two Banach spaces $\mathscr{E}$ and $\mathscr{E}^{\prime}: \mathscr{E}$ is the space of translationally invariant real potential with norm:

$$
\||\Phi|\|=\sum_{X \ni 0} \frac{\operatorname{diam} X+1}{|X|}|\Phi(X)|<\infty .
$$

$\mathscr{E}^{\prime}$ is the larger space of complex potentials with norm

$$
\|\Phi\|\left|=\sum_{X \ni 0} \frac{\operatorname{diam} X+1}{|X|} \sup _{t \in \mathbb{Z}}\right| \Phi(X+t) \mid .
$$


In this paper we will show that the infinite volume correlation functions vary analytically with the interaction parameters. This problem was already solved by Dobrushin [1] using sophisticated estimates of mean values of exponential functionals of random processes. We present a new proof based on a specific renormalization group transformation (the so-called decimation [2]) and the cluster expansion [3-6]. For the sake of simplicity we state our results for the pressure; but the reader could easily work out the analogous properties for the correlation functions by slightly modifying the proof.

Theorem. Let $\Phi \in \mathscr{E}$ and $\Psi_{i} \in \mathscr{E}^{\prime}$ with $\left\|\Psi_{i}\right\|=1 \quad i=1,2, \ldots, d$ and $\lambda \equiv\left(\lambda_{i}, \ldots, \lambda_{d}\right) \in \omega$, where $\omega$ is a suitable sphere in $\mathbb{C}^{d}$ centered at the origin. Then the limit

$$
F\left(\Phi+\sum_{i=1}^{d} \lambda_{i} \Psi_{i}\right)=\lim _{\Lambda>\mathbb{Z}|\Lambda|} \frac{1}{\log }\left(\sum_{x_{\Lambda}} \exp \left[-H_{\Lambda}\left(x_{\Lambda}\right)\right]\right)
$$

exists and is holomorphic in $\lambda$ on $\omega$.

The proof is given in Sect. 2 ; in the sequel we will briefly sketch the general strategy of our approach. We start by grouping the sites of the lattice in alternating blocks of different sizes $L$ and $M$ with, say, $M \geqq L$. It follows from (1.2) that

i) The interaction among consecutive blocks satisfies a bound uniform in $L$ and $M$.

ii) The interaction among more than two consecutive blocks or any number of nonconsecutive blocks goes to zero as $L$ and $M \rightarrow \infty$.

If we call $\alpha_{i}\left(\beta_{i}\right)$ the configurations in a block of size $L(M)$, the partition function of the system can be written in the following way

$$
\begin{aligned}
Z= & \sum_{\alpha} \prod_{i} e^{a\left(\alpha_{i}\right)} \prod_{j} \sum_{\beta_{j}} \exp \left[J\left(\beta_{j}, \alpha_{j}, \alpha_{j+1}\right)\right] \exp \left[\sum W(\boldsymbol{\alpha})\right] \\
& \cdot \sum_{\boldsymbol{\beta}}\left\{\prod_{j}\left[\exp \left[J\left(\beta_{i}, \alpha_{i} \alpha_{i+1}\right)\right] / \sum_{\beta_{j}} \exp \left[J\left(\beta_{j} \alpha_{j} \alpha_{j+1}\right)\right]\right]\right. \\
& \left.\cdot \exp \left[\sum W(\boldsymbol{\alpha}, \boldsymbol{\beta})\right]\right\},
\end{aligned}
$$

where, roughly speaking, $J$ contains the interactions among nearest neighbor blocks and $W$ the tails of the potential and perturbations which are small for $\lambda \in \omega$.

For a precise definition of the various terms see (2.1)-(2.3).

The next step is to perform the averages over the $\beta$ 's (i.e. the $x_{t}$ 's in the blocks of size $M$ ), so that we are left with an effective interaction among the $\alpha$ 's where we can distinguish three different kinds of contributions:
a) $\sum W(\boldsymbol{\alpha})$
b) $\sum_{i} \log \left[\sum_{\beta_{i}} J\left(\beta_{i} \alpha_{i}, \alpha_{i+1}\right)\right]$
c) $\log \left\{\sum_{\boldsymbol{\beta}} \prod\left[\exp J / \sum \exp J\right] \cdot \exp \left[\sum W(\boldsymbol{\alpha} \boldsymbol{\beta})\right]\right\}$.

The terms in a) can always be made as small as we please for $M$ sufficiently large [cf. ii)]. If we subtract from b) the one body contributions, the resulting two body potentials go to zero when $M \rightarrow \infty$ (see Lemma 3). The quantity in c) can be 
thought as the log of the partition function of a spin system on a lattice where on each site a spin of size $2^{M}$ sits, and where the interactions among different sites can be made very small for $L$ sufficiently large uniformly in $M$.

A standard way to study these systems is by a cluster expansion that allows us to express the log of the partition function as a convergent sum of local terms. We make estimates (see Appendix A and B) allowing us to apply the general formalism to our case. We show not only that the contributions from c) to the effective interaction go to zero for $L \rightarrow \infty$, uniformly in $M$, but also that they depend analytically on the $\lambda$ 's in a suitable neighborhood of the origin (cf. Lemma 2).

In conclusion we obtain, by the decimation procedure, an effective interaction among the $\alpha$ 's that for $L$ and $M$ sufficiently large is as small as we please.

\section{Proof of the Theorem}

Let $L, M$, and $p$ be three positive integers, $L$ and $M$ are both odd and $p$ is arbitrary, $\Lambda_{p}$ is the interval centered at the origin: $\left|\Lambda_{p}\right|=2 p L+(2 p+1) M$. Consider now the decomposition of $\Lambda_{p}$ into consecutive blocks $B_{-p}, A_{-p+1}, B_{-p+1}, \ldots, B_{p-1}, A_{p}, B_{p}$ where $\left|A_{i}\right|=L$ and $\left|B_{i}\right|=M$.

Let $\Gamma_{p}^{B}$ be the set of all $A$ blocks contained in $\Lambda_{p}: \Gamma_{p}^{A}=\left\{A_{i}, i=-p+1, \ldots, p\right\}$ and analogously for the $B$-blocks : $\Gamma_{p}^{B}=\left\{B_{i}, i=-p, \ldots, p\right\}$. The decomposition of $\Lambda_{p}$ in $A$ and $B$ blocks in the limit $p \rightarrow \infty$ induces an analogous decomposition in $\mathbb{Z}$. $\Gamma_{\infty}^{A}\left(\Gamma_{\infty}^{B}\right)$ is the set of all $A$-blocks ( $B$-blocks) contained in $\mathbb{Z}$.

Call $\alpha_{i}=x_{A_{i}}$ and $\beta_{i}=x_{B_{i}}$ the configurations in the blocks $A_{i}$ and $B_{i}$ and set $\alpha_{i}$ $=\mathbf{0} \equiv(0, \ldots, 0, \ldots, 0)$. For $V \subset \Gamma_{\infty}^{A} \cup \Gamma_{\infty}^{B}$ we denote by $\beta_{V}\left(\alpha_{V}\right)$ the configuration in $V \cap \Gamma_{\infty}^{B}\left(V \cap \Gamma_{\infty}^{A}\right)$. More precisely:

$$
\beta_{V}=\left\{x_{t}, t \in \bigcup_{A_{i} \subset V \cap \Gamma_{\infty}^{A}} A_{i}\right\}, \quad \alpha_{V}=\left\{x_{t}, t \in \bigcup_{B_{i} \subset V \cap \Gamma_{\infty}^{B}} B_{i}\right\},
$$

we still use the notation $\alpha_{i}$ for $\alpha_{A_{i}}\left(\beta_{i}\right.$ for $\beta_{A_{i}}$ ). Now rewrite the Hamiltonian (1.1) so that the interactions among blocks are made explicit

$$
\begin{aligned}
& -H_{\Lambda_{p}}^{\Phi}\left(x_{\Lambda_{p}}\right)=\sum_{i=-p+1}^{p} a_{i}^{\Phi}\left(\alpha_{i}\right)
\end{aligned}
$$

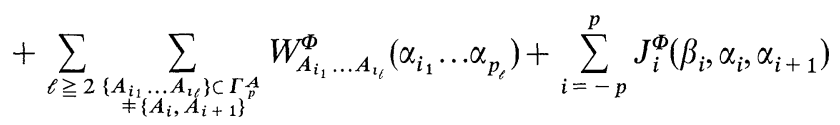

$$
\begin{aligned}
& +\sum_{\substack{\ell \geqq 1 \\
q \geqq 0}} \sum_{\left\{B_{i_{1}} \ldots B_{\ell_{\ell}}, A_{j_{1}} \ldots A_{J_{q}}\right\} \bar{\Gamma}_{p}} W_{B_{i_{1}} \ldots B_{i_{\ell}}, A_{j_{1}} \ldots A_{j_{q}}}^{\Phi}\left(\beta_{i_{1}} \ldots \alpha_{j_{q}}\right)
\end{aligned}
$$

with $\alpha_{-p}=\alpha_{p+1}=\mathbf{0} . \bar{\Gamma}_{p}$ consists of all the sets $C=A_{j_{1}} \ldots A_{j_{q}}, B_{i_{1}} \ldots B_{i_{\ell}} ; A_{i_{k}} \in \Gamma_{p}^{\boldsymbol{A}}$, $B_{i_{k}} \in \Gamma_{p}^{B}$ such that

$$
\begin{gathered}
C \cap \Gamma_{p}^{B} \neq \emptyset, \quad C \neq A_{i} B_{i} A_{i+1}, \quad C \neq A_{i} B_{i}, \\
C \neq B_{i} A_{i+1}, \quad C \neq B_{i},
\end{gathered}
$$


namely $\bar{\Gamma}_{p}$ collects all the sets of $A$ and $B$ blocks which are not considered in $J$

$$
\begin{aligned}
& a_{i}^{\Phi}\left(\alpha_{i}\right)=\sum_{X \subset A_{i}} \Phi(X) \prod_{t \in X} x_{t} \\
& W_{B_{i_{1}} \ldots B_{\ell_{\ell}}, A_{j_{1}} \ldots A_{j_{q}}}^{\Phi}\left(\beta_{i_{1}} \ldots \alpha_{j_{q}}\right)=\sum_{\emptyset \neq X_{i_{1}} \subset B_{i_{1}} \ldots \emptyset \neq Y_{j_{q}} \in A j_{q}} \Phi\left(\left(\bigcup_{k=1}^{\ell} X_{i_{k}}\right) \cup\left(\bigcup_{h=1}^{q} Y_{j_{q}}\right)\right) \\
& \cdot \prod_{k=1}^{\ell} \prod_{t \in X_{i_{k}}} x_{t} \prod_{h=1}^{q} \prod_{t \in Y_{j_{h}}} x_{t} \\
& J_{i}^{\Phi}\left(\beta_{i} \alpha_{i} \alpha_{i+1}\right)=\sum_{\substack{X \subset A_{i-1} \cup B_{i} \cup A_{\imath} \\
X \cap B_{i} \neq \emptyset}} \Phi(X) \prod_{t \in X} x_{t}+W_{A_{\imath-1}, A_{i}}^{\Phi}\left(\alpha_{i-1}, \alpha_{i}\right) .
\end{aligned}
$$

Consider now a potential of the form $\Phi+\Psi_{\lambda}$ with $\Psi_{\lambda}=\sum_{i=1}^{d} \lambda_{i} \Psi_{i}$ where $\Phi \in \mathscr{E}$, $\Psi_{i} \in \mathscr{E}^{\prime}$ with $\left\|\Psi_{i}\right\|=1, i=1, \ldots, d$ and $\lambda \equiv\left(\lambda_{1}, \ldots, \lambda_{d}\right) \in \mathbb{C}^{d}$ and define the effective Hamiltonian obtained via the decimation transformation by

$$
\begin{aligned}
& \exp \left(-\bar{H}_{\Lambda_{p}}^{\Phi+\Psi_{\lambda}}\left(\alpha_{\Gamma_{p}^{A}}\right)\right)=\exp \left\{\sum_{i=-p+1}^{p} a_{i}^{\Phi+\Psi_{\lambda}}\left(\alpha_{i}\right)\right. \\
& \left.+\sum_{\ell \geqq 2} \sum_{\left\{A_{i_{1}} \ldots A_{t_{\ell}}\right\} \neq\left\{A_{i} A_{i+1}\right\}} W_{A_{i_{1}} \ldots A_{t_{\ell}}}^{\Phi+\Psi_{\lambda}}\left(\alpha_{i_{1}}, \ldots, \alpha_{i_{\ell}}\right)\right\} \\
& \cdot \sum_{\beta_{\Gamma_{p}^{\mathrm{B}}}} \exp \left\{\sum_{i=-p}^{p} J_{i}^{\Phi}\left(\beta_{i}, \alpha_{i}, \alpha_{i+1}\right)+J_{i}^{\Psi \lambda}\left(\beta_{i}, \alpha_{i}, \alpha_{i+1}\right)\right. \\
& \left.+\sum_{\substack{\ell \geqq 1 \\
q \geqq 0}} \sum_{\left\{B_{i_{1}} \ldots A_{j_{q}}\right\} \in \Gamma_{p}} W_{B i_{1} \ldots \hat{A}_{j_{q}}}^{\Phi+\Psi_{\lambda_{1}}}\left(\beta_{i_{1}}, \ldots, \alpha_{j_{q}}\right)\right\},
\end{aligned}
$$

or in more compact form

$$
\begin{aligned}
& -\bar{H}_{A_{p}}^{\Phi+\Psi_{\lambda}}\left(\alpha_{\Gamma_{p}^{A}}\right)=\sum_{i=-p+1}^{p} a_{i}^{\Phi+\Psi_{\lambda}}\left(\alpha_{i}\right) \\
& +\sum_{\ell \geqq 2\left\{A_{i_{1}} \ldots A_{i_{\ell}}\right\} \neq\left\{A_{i} A_{i+1}\right\}} W_{A_{i_{1}} \ldots \hat{A}_{\lambda_{\ell}}}^{\Phi+\Psi_{\lambda_{i}}}\left(\alpha_{i_{1}} \ldots \alpha_{i_{\ell}}\right) \\
& +\sum_{i=-p}^{p} \log Z_{B_{i}}^{\alpha_{i}, \alpha_{i+1}}(\Phi)+\tilde{H}_{A_{p}}^{\Phi+\Psi_{\lambda}}\left(\alpha_{\Gamma_{p}^{A}}\right)
\end{aligned}
$$

with

$$
\begin{gathered}
Z_{B_{i}}^{\alpha_{i}, \alpha_{i+1}}(\Phi)=\sum_{\beta_{i}} \exp \left\{J_{i}^{\Phi}\left(\beta_{i}, \alpha_{i}, \alpha_{i+1}\right)\right\} \\
-\tilde{H}_{\Lambda_{p}}^{\Phi+\Psi_{\lambda}}\left(\alpha_{\Gamma_{P}^{A}}\right)=\log \left\{\sum_{\beta_{\Gamma_{p}}^{B} i=-p+1} \prod_{\beta_{i}}^{p}\left[\frac{\exp \left(J_{i}^{\Phi}\left(\beta_{i} \alpha_{i} \alpha_{i+1}\right)\right)}{\sum_{i} \exp \left(J_{i}^{\Phi}\left(\beta_{i} \alpha_{i} \alpha_{i+1}\right)\right)}\right]\right\} \\
\cdot \prod_{C \in \bar{\Gamma}_{p}} \exp \left[W_{C}^{\Phi+\Psi_{\lambda}}\left(\alpha_{C} \beta_{C}\right)\right] \prod_{i=-p}^{p} \exp \left[J_{i}^{\Psi \lambda}\left(\beta_{i} \alpha_{i} \alpha_{i+1}\right)\right] .
\end{gathered}
$$


Definition 1 . Given an arbitrary set $G$, let $\tilde{G}$ be an arbitrary subset of $G$ and $\mathscr{G}$ the collection of all finite subsets of $G$. We will call any $C_{i} \in \mathscr{G}$ a bond. $\tilde{C}_{i}$ will denote the union of the elements of $\tilde{G}$ belonging to $C_{i}$.

Definition 2. A set of bonds $C_{1}, \ldots, C_{k} \in \mathscr{G}, \tilde{C}_{i} \neq \emptyset$, is a polymer $R$ if for any choice of $C_{t} ; C_{s} \in R \exists C_{i_{1}} \ldots C_{i_{r}} \in R$ with $C_{i_{1}}=C_{t}, C_{i_{r}}=C_{s}$ and $\tilde{C}_{i_{h}} \cap \tilde{C}_{i_{h+1}} \neq \emptyset-h=1, \ldots, r-1$.

Let $\mathscr{R}$ be the set of all polymers. For $R=C_{1}, \ldots, C_{k}$, let $\tilde{R}=\bigcup_{i=1}^{k} \tilde{C}_{i}, \bar{R}=\bigcup_{i=1}^{k} C_{i}$. The activity is a function $\zeta: \mathscr{R} \rightarrow \mathbb{C}$. A system of polymers in $F \subset G$ with activity $\zeta$ and hard core interaction is described by the partition function:

$$
\Xi_{F}(\zeta)=1+\sum_{n \geqq 1} \sum_{\substack{R_{1} \ldots R_{n} \\ \tilde{R}_{\imath} \subset F, \tilde{R}_{\imath} \cap \tilde{R}_{j}=0}} \prod_{i=1}^{n} \zeta\left(R_{i}\right) .
$$

Lemma 1. Given a polymer system, if the activities are such that

$$
|\zeta(R)| \leqq \sigma^{|\tilde{R}|} \prod_{C \in R} \varphi_{C}
$$

with

$$
0<\sigma<1, \quad \varphi_{C}>0, \quad \sup _{a \in \tilde{G}} \sum_{C \ni a} \varphi_{C} \leqq K
$$

then, when

$$
\exp K<[\sqrt{\sigma}(2-\sqrt{\sigma})]^{-1}
$$

the following relations hold:

$$
\begin{gathered}
\sup _{a \in \tilde{\boldsymbol{G}}} \sum_{R: \tilde{\tilde{R}} \ni a}|\zeta(R)| \leqq \frac{K \sigma}{1-\frac{\sigma(\exp K-1)}{(1-\sigma \exp K)(1-\sigma)}} \equiv G(K, \sigma), \\
\sum_{n \geqq 1} \sum_{\substack{R_{1} \ldots R_{n}: \\
\exists R_{i}=R}}\left|\varphi_{T}\left(R_{1} \ldots R_{n}\right)\right| \prod_{i=1}^{n}\left|\zeta\left(R_{i}\right)\right| \\
\leqq \mid \zeta(R) \frac{\exp [G(K, \sqrt{\sigma})|\tilde{R}|]}{1-\sqrt{\sigma} \exp [G(K, \sqrt{\sigma})]}
\end{gathered}
$$

with

$$
\varphi_{T}\left(R_{1}, \ldots, R_{n}\right)=\frac{1}{n !} \sum_{g \in \mathscr{G}_{n}\left(R_{1} \ldots R_{n}\right)}(-1)^{\#(\text { edges in } g)},
$$

where $\mathscr{G}_{n}\left(R_{1} \ldots R_{n}\right)$ is the set of connected graphs with $n$ vertices $(1, \ldots, n)$ and edges $(i, j)$ corresponding to pairs $R_{i}, R_{j}$ such that $\tilde{R}_{i} \cap \tilde{R}_{j} \neq \emptyset$. We set the sum equal to zero if $\mathscr{G}_{n}$ is empty and one if $n=1$ and finally

$$
\Xi_{F}(\zeta)=\exp \left[\sum_{n \geqq 1} \sum_{\substack{R_{1} \ldots R_{n} \\ R_{i} \subset \boldsymbol{F}}} \varphi_{T}\left(R_{1} \ldots R_{n}\right) \prod_{i=1}^{n} \zeta\left(R_{i}\right)\right] .
$$


Proof. (2.9) and (2.10) can be deduced by standard methods from (2.8) [3]. The proof of (2.8) is given in Appendix $B$.

Lemma 2. Set $G=\Gamma_{\infty}^{A} \cup \Gamma_{\infty}^{B}, \tilde{G}=\Gamma_{\infty}^{B}$ and define the activities of the associated polymers in the following way:

For

$$
\begin{gathered}
R=C_{1}, \ldots, C_{k} \quad \text { with } \begin{array}{r}
C_{i} \in \bar{\Gamma}_{\infty} \text { for }\left|C_{i}\right|>1, \\
C_{i} \in \Gamma_{\infty}^{B} \text { for }\left|C_{i}\right|=1,
\end{array} \\
\zeta_{\lambda}(R)=\sum_{\beta_{\tilde{R}}} \prod_{B_{i} \in \tilde{R}} \frac{\exp \left[J_{i}^{\Phi}\left(\beta_{i}, \alpha_{i}, \alpha_{i+1}\right)\right]}{\sum_{\beta_{i}} \exp \left[J_{i}^{\Phi}\left(\beta_{i}, \alpha_{i}, \alpha_{i+1}\right)\right]} \\
\cdot \prod_{s=1}^{k}\left[\exp U_{C_{s}}\left(\alpha_{C_{s}}, \beta_{C_{s}}\right)-1\right],
\end{gathered}
$$

where $U_{B_{i}}=J_{i}^{\Psi \lambda}\left(\beta_{i} \alpha_{i} \alpha_{i+1}\right)$ and for $C \neq B_{i}, C \in \bar{\Gamma}_{\infty}, U_{C}\left(\alpha_{C}, \beta_{C}\right)=W_{C}^{\Phi+\Psi_{\lambda}}\left(\alpha_{C}, \beta_{C}\right)$. For the remaining polymers $\zeta(R)=\mathbf{0}$.

Then $\exists L_{0}$ such that $\forall M$ and for $\lambda$ varying in a sufficiently small sphere $\omega_{M}$ whose

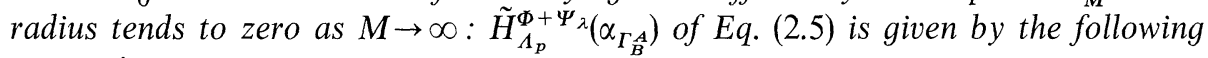
expression

$$
\begin{gathered}
-\tilde{H}_{\Lambda_{p}}^{\Phi+\Psi_{\lambda}}\left(\alpha_{\Gamma_{B}^{A}}\right)=\sum_{D \subset \Gamma_{B}^{A}} \tilde{W}^{\Phi+\Psi_{\lambda}}\left(\alpha_{D}\right), \\
\tilde{W}^{\Phi+\Psi_{\lambda}}\left(\alpha_{D}\right)=\sum_{R_{1} \ldots R_{n}} \varphi_{T}\left(R_{1} \ldots R_{n}\right) \cdot \prod_{i=1}^{n} \zeta_{\lambda}\left(R_{i}\right),
\end{gathered}
$$

where $\sum^{D}$ is a sum over all the clusters of polymers such that $\prod_{i=1}^{n} \zeta\left(R_{i}\right)$ is a function of all the variables $\alpha_{i_{1}} \ldots \alpha_{i_{|D|}}$ but does not depend on any other $\alpha$. Moreover $\forall \Lambda_{p}, D \subset \Lambda_{p}$, $\forall \alpha_{D}$. $\tilde{W}_{D}^{\Phi+\Psi_{\lambda}}\left(\alpha_{D}\right)$ is a holomorphic function of $\lambda$ in $\omega_{M}$.

Proof. Setting $G=\Gamma_{\infty}^{A} \cup \Gamma_{\infty}^{B}, \tilde{G}=\Gamma_{\infty}^{B}$, it is easy to check that

$$
\exp \left[-\tilde{H}_{A_{p}}^{\Phi+\Psi}\left(\alpha_{\Gamma_{p}^{A}}\right)\right]=1+\sum_{n \geqq 1} \sum_{\substack{R_{1} \ldots R_{n} \\ \bar{R}_{2} \subset \Gamma_{p}^{A} \cup \Gamma_{p}^{B} \\ \tilde{R}_{l} \cap \tilde{R}_{J}=\emptyset}} \prod_{i=1}^{n} \zeta_{\lambda}\left(R_{i}\right) .
$$

In Appendix A we will show that for $R=C_{1}, \ldots, C_{k}, B_{i_{1}}, \ldots, B_{i_{h}}$,

$$
\begin{gathered}
C_{s} \in \bar{\Gamma}_{p} \quad s=1, \ldots, k, \quad \ell=1, \ldots, h \\
\left|\zeta_{\lambda}(R)\right| \leqq \varrho^{|\tilde{R}|} \prod_{s=1}^{k} 2\left\|W_{C_{s}}^{\Phi+\Psi_{\lambda}}\right\| \prod_{\ell=1}^{h} 2\left\|J_{i}^{\Psi_{\lambda}}\right\|,
\end{gathered}
$$

where

$$
\varrho=(1+\exp (-\|\Phi\| \|))^{-1},
$$


and we denote by $\|f\|$ the sup norm of the continuous function $f$. We have:

$$
\sup _{i} \sum_{C \ni B_{i}}\left\|\mathrm{~W}_{C}^{\Phi+\Psi_{\lambda}}\right\|=\varepsilon(\mathrm{L}) \underset{L \rightarrow \infty}{\longrightarrow} \mathrm{O}
$$

uniformly in $M$.

Equation (2.15) is an immediate consequence of the fact that for the potentials belonging to $\mathscr{E}$ [see Eq. (1.2)], the interaction between two consecutive halflines is finite. Furthermore if $\lambda \in \omega_{M}$, i.e. $\lambda$ :

$$
\sup _{i=1, \ldots, d}\left|\lambda_{i}\right| \leqq \frac{1}{4 M d} \min \left[1, \log (\sqrt{\sigma}(2-\sqrt{\sigma}))^{-1}\right],
$$

it is easily seen that for $L$ sufficiently large the conditions of Lemma 1 are satisfied and (2.12) holds.

Since each $\zeta_{\lambda}(R)$ is an analytic function of $\lambda$ and the series $\left(2.12^{\prime}\right)$ is absolutely and uniformly convergent for $\lambda \in \omega_{M}$, it follows that $\forall \Lambda_{p}, \forall D \subset \Lambda_{p}, \forall \alpha_{D}, \tilde{W}_{D}^{\Phi+\Psi_{\lambda}}\left(\alpha_{D}\right)$ is analytic for $\lambda \in \omega_{M}$.

Lemma 3. $\forall L<\infty$

$$
\lim _{M \rightarrow \infty} \sup _{\alpha_{i}, \alpha_{i+1}} \log \left[\frac{Z_{\boldsymbol{B}_{i}}^{\alpha_{i}, \alpha_{i+1}}(\Phi) \cdot Z_{\boldsymbol{B}_{i}}^{0,0}(\Phi)}{Z_{B_{i}}^{\alpha_{i}, 0}(\Phi) \cdot Z_{\boldsymbol{B}_{i}}^{0, \alpha_{i+1}}(\Phi)}\right]=0 .
$$

Proof. The proof, a simple application of Theorem 3 of [8], is given in Appendix C.

Proof of the Theorem. Using definitions (2.1), (2.3), and (2.3') we get:

$$
\begin{aligned}
Z_{\Lambda_{p}}\left(\Phi+\Psi_{\lambda}\right)=\sum_{x_{\Lambda_{p}}} \exp \left[-H_{\Lambda_{p}}^{\Phi+\Psi_{\lambda}}\left(x_{\Lambda_{p}}\right)\right. \\
=\sum_{\alpha_{\Gamma_{p}^{A}}} \exp \left[-\bar{H}_{\Lambda_{p}}^{\Phi+\Psi_{\lambda}}\left(\alpha_{\Gamma_{p}^{A}}\right)\right]=\prod_{i=-p+1}^{p} \sum_{\alpha_{i}} \exp \left[\bar{a}^{\Phi}\left(\alpha_{i}\right)\right] \\
\quad \cdot \sum_{\alpha_{\Gamma_{p}^{A}}} \prod_{i=}^{p} \frac{\exp \left[\bar{a}^{\Phi}\left(\alpha_{i}\right)\right]}{\sum_{\alpha_{l}} \exp \left[\bar{a}^{\Phi}\left(\alpha_{\imath}\right)\right]} \prod_{D \subset \Gamma_{p}^{A}} \exp \left[V_{D}\left(\alpha_{D}\right)\right]
\end{aligned}
$$

where

$$
\begin{gathered}
\bar{a}^{\Phi}\left(\alpha_{i}\right)=a^{\Phi}\left(\alpha_{i}\right)+\log \frac{Z_{B_{i}}^{\alpha_{i}, 0}(\Phi)}{Z_{B_{i}}^{0,0}(\Phi)}+\log \frac{Z_{B_{i-1}}^{0, \alpha_{2}}(\Phi)}{Z_{B_{i-1}}^{0,0}(\Phi)} \\
V_{A_{i}}\left(\alpha_{i}\right)=a_{i}^{\Psi \lambda}\left(\alpha_{i}\right) \\
V_{A_{2} A+1}\left(\alpha_{i} \alpha_{i+1}\right)=\tilde{W}_{A_{i}, A_{i+1}}^{\Psi_{\lambda}}\left(\alpha_{i}, \alpha_{i+1}\right) \\
+\log \left[\frac{Z_{B_{i+1}}^{\alpha_{2}, \alpha_{i+1}}(\Phi) \cdot Z_{B_{i+1}}^{0,0}(\Phi)}{Z_{B_{i+1}, 0}^{\alpha_{2}, 0}(\Phi) \cdot Z_{B_{i+1}}^{0, \alpha_{2}}(\Phi)}\right]
\end{gathered}
$$

and for $A_{i_{1}} \ldots A_{i \ell} \neq A_{i} A_{i+1}, A_{i}$

$$
V_{A_{i_{1}} \ldots A_{i_{\ell}}}\left(\alpha_{i_{1}} \ldots \alpha_{i_{\ell}}\right)=W_{A_{i_{1}} \ldots A_{i_{\ell}}}^{\Phi+\Psi_{\lambda}}\left(\alpha_{i_{1}} \ldots \alpha_{i_{\ell}}\right)+\tilde{W}_{A_{i_{1}} \ldots A_{i_{\ell}}}^{\Phi+\psi_{\lambda}}\left(\alpha_{i_{1}} \ldots \alpha_{i_{\ell}}\right)
$$


Now setting $G=\Gamma_{\infty}^{A}=\tilde{G}$ (see Definition 1) and

$$
\Theta_{\lambda}(S)=\sum_{\alpha \tilde{s}} \prod_{A_{i} \in \tilde{S}} \frac{\exp \left[\bar{a}^{\Phi}\left(\alpha_{i}\right)\right]}{\sum_{\alpha_{i}} \exp \left[\bar{a}^{\Phi}\left(\alpha_{i}\right)\right]} \prod_{D \in S}\left(\exp V_{D}-1\right),
$$

we get

$$
\begin{aligned}
Z_{\Lambda_{p}}\left(\Phi+\Psi_{\lambda}\right)= & \prod_{i=}^{p} \sum_{p+1} \sum_{\alpha_{i}} \exp \left[\bar{a}^{\Phi}\left(\alpha_{i}\right)\right] \\
& \cdot\left(1+\sum_{n \geqq 1} \sum_{\left\{S_{1} \ldots S_{n}\right\}} \prod_{i=1}^{n} \Theta_{\lambda}\left(S_{i}\right)\right)
\end{aligned}
$$

Using Appendices A and B we obtain that

$$
\sup _{i} \sum_{S \ni A_{i}}\left|\Theta_{\lambda}(S)\right| \leqq G\left[K(\lambda, M, L), \varrho^{1 / 4}\right]
$$

when

$$
K(\lambda, M, L) \leqq \log \left[\frac{1}{\varrho^{1 / 4}\left(2-\varrho^{1 / 4}\right)}\right],
$$

with $\varrho=[1+\exp (-\|\Phi\|)]^{-1}$ and

$$
\begin{aligned}
& K(\lambda, L, M) \leqq \sup _{A \in \Gamma_{\infty}^{A}} \sum_{D \ni A}\left\{2\left\|W_{D}^{\Phi+\Psi_{\lambda}}\right\|\right. \\
& \left.\quad+\sum_{n \geqq 1} \sum_{R_{1} \ldots R_{n}}^{D}\left|\varphi^{T}\left(R_{1} \ldots R_{n}\right)\right| \prod_{i=1}^{n}\left(\frac{1}{\sqrt{\varrho}}\right)^{\left|\tilde{R}_{i}\right|}\left\|\zeta\left(R_{i}\right)\right\|\right\} \\
& \quad+\sup _{i} \frac{2}{\sqrt{\varrho}}\left\|a_{i}^{\Psi_{\lambda}}\right\|+\frac{2}{\varrho} \sup _{\alpha_{0} \alpha_{1}}\left|\log \left[\frac{Z_{B_{0}}^{\alpha_{0}, \alpha_{1}}(\Phi) \cdot Z_{B_{0}}^{0,0}(\Phi)}{Z_{B_{0}}^{\alpha_{0}, 0}(\Phi) \cdot Z_{B_{0}}^{0, \alpha_{1}}(\Phi)}\right]\right|
\end{aligned}
$$

Notice that the bound (2.22) is independent of $p$. Now, $\forall \bar{K}>0$ we have

i) $\forall \Phi \in \mathscr{E}$ and $\forall L$ it follows from Lemma 3 that $\exists M_{0}(L): \forall M \geqq M_{0}(L)$

$$
\stackrel{2}{\varrho} \sup _{\alpha_{0} \alpha_{1}}\left|\log \left[\frac{Z_{B_{0}}^{\alpha_{0}, \alpha_{1}}(\Phi) \cdot Z_{B_{0}}^{\alpha_{0}, \alpha_{1}}(\Phi)}{Z_{B_{0}}^{\alpha_{0}, 0}(\Phi) \cdot Z_{B_{0}}^{0, \alpha_{1}}(\Phi)}\right]\right|<\frac{\bar{K}}{3},
$$

ii) from the proof of Lemma 2 it follows that $\exists L_{0}: \forall L \geqq L_{0}, \forall M, \forall \lambda \in \omega_{M}$

$$
\sup _{A \in \Gamma_{\infty}^{A}} \sum_{D \ni A} \sum_{R_{1} \ldots R_{n}}^{D}\left|\varphi^{T}\left(\mathrm{R}_{1} \ldots \mathrm{R}_{n}\right)\right| \prod_{i=1}^{n}\left(\frac{1}{\sqrt{\varrho}}\right)^{\left|\tilde{R}_{i}\right|}\left|\zeta_{\lambda}\left(\mathrm{R}_{i}\right)\right|<\frac{\bar{K}}{3},
$$

iii) for $L=L_{0} \exists L_{1}\left(L_{0}\right): \forall \lambda \in \omega_{L_{1}\left(L_{0}\right)}, \forall M \geqq L_{1}\left(L_{0}\right)$

$$
\sup _{i} \frac{2}{\sqrt{\varrho}}\left\|a_{i}^{\Psi_{\lambda}}\right\|+\sup _{A \in \Gamma_{\infty}^{A}} \sum_{D \ni A} 2\left\|W_{D}^{\Phi+\Psi_{\lambda}}\right\|<\frac{\bar{K}}{3} .
$$

Then for $\lambda \in \omega_{\bar{M}}$ where $\bar{M}=\max \left[L_{1}\left(L_{0}\right), M_{0}\left(L_{0}\right)\right]$ we have $K\left(\lambda, L_{0}, \bar{M}\right)<\bar{K}$. Now if

$$
\bar{K}<\min \left[\frac{1}{2}, \log \left(\varrho^{1 / 8}\left(2-\varrho^{1 / 8}\right)\right)^{-1}\right]
$$


from (2.8), (2.9) we see that for $L=L_{0}, M=\bar{M}$

$$
\begin{aligned}
& \sup _{\lambda \in \omega_{\bar{M}}}\left|\Lambda_{p}\right|^{-1}\left|\log Z_{\Lambda_{p}}\left(\Phi+\Psi_{\lambda}\right)\right| \\
& \leqq \sup _{\lambda \in \omega_{\bar{M}}} \sup _{i} \sum_{S_{\ni A_{i}}} \sum_{\substack{S_{1} \ldots S_{S_{n}} \\
\exists S_{j}}}\left|\varphi_{T}\left(S_{1} \ldots S_{n}\right)\right| \prod_{i=1}^{n}\left|\Theta_{\lambda}^{p}\left(S_{\imath}\right)\right| \\
& \quad+\frac{1}{M} \log \left[\sum_{\alpha} \exp \left(\bar{a}^{\Phi}(\alpha)\right)\right] \leqq \frac{G\left(\bar{K}, \varrho^{1 / 8}\right)}{1-\varrho^{1 / 8} G\left(\bar{K}, \varrho^{1 / 8}\right)} \\
& \quad+\frac{1}{M} \log \left[\sum_{\alpha} \exp \left(\bar{a}^{\Phi}(\alpha)\right)\right] .
\end{aligned}
$$

For real $\Phi$ the existence of the limit

$$
\lim _{\Lambda_{p}>\mathbb{Z}} \frac{\log Z_{\Lambda_{p}}(\Phi)}{\left|\Lambda_{p}\right|}
$$

is a standard result. Then from the uniform bound (2.28), using Vitali's theorem we conclude the proof.

\section{Conclusions}

In the previous sections we have discussed only the case of a lattice gas, but our approach has a much wider validity. For instance if we consider a continuous system of particles with hard core and finite first moment interactions, then under very reasonable smoothness conditions for the potentials the theorem given in Sect. 1 holds. In fact if we consider cells of size $a$ (where $a$ is the hard core range) and construct alternating blocks of size $L a$ and $M a$, we can repeat the same steps of Sect. 2, where now the analog of Lemma 3 will be a consequence of Lemma 6 of [8] and the analog of Appendix A can be easily obtained if the long range potential is uniformly bounded.

To extend these results to more general systems we think it worthwhile to remark that if we consider a potential $\Phi \in \mathscr{E}$ which, for some positive $r$ makes the norm $\|\Phi\|_{r}=\sum_{X \ni 0} e^{r|X|}|\Phi(X)|$ finite, then the proof given in the previous section is greatly simplified because:

a) An estimate analogous to (2.14) is trivially obtained for $\sigma=e^{-r}$.

b) The effective potential $V_{D}\left(\alpha_{D}\right)$ for $L$ and $M$ sufficiently large is such that the norm

$$
\left\|V\left|\|_{r / 4}=\sup _{A \in \Gamma_{\infty}^{A}} \sum_{D \ni A} e^{r / 4|D|} \sup _{\alpha_{D}}\right| V_{D}\left(\alpha_{D}\right) \mid\right.
$$

can be made small enough in order to apply Isreal's analiticity theorem [9]. So that, assuming the analog of Lemma 3, our theorem holds also for a bounded spin lattice system where the potential $\Phi$ is such that for some $r>0$

$$
\sum_{X \ni 0} e^{r|X|}(\operatorname{diam} X+1)\left\|\Phi_{X}\right\|<\infty
$$


The above discussion suggests the conjecture that our approach should be valid also for unbounded spins and continuous systems of particles, without hard core, if some suitable superstability [10] conditions are satisfied.

\section{Appendix A}

We have, for $R=C_{1} \ldots C_{k}, B_{i_{1}} \ldots B_{i_{h}} ; C_{s} \in \bar{\Gamma}_{\infty}, B_{i_{f}} \in \Gamma_{\infty}^{B}$,

$$
\begin{aligned}
& \zeta_{\lambda}(R)=\sum_{\beta_{\tilde{R}}} \prod_{i \in \tilde{R}}\left[\frac{\exp \left[J^{\Phi}\left(\beta_{i}, \alpha_{i}, \alpha_{i+1}\right)\right]}{\sum_{\beta_{\imath}} \exp \left(J^{\Phi}\left(\beta_{i}, \alpha_{i}, \alpha_{i+1}\right)\right)}\right] \\
& \cdot \prod_{s=1}^{k}\left[\exp \left(W_{C_{s}}^{\Phi+\Psi_{\lambda}}\left(\alpha_{C_{s}}, \beta_{C_{s}}\right)\right)-1\right] \prod_{\ell=1}^{h}\left[\exp \left(J_{i_{\iota}}^{\Psi_{\iota}}\left(\beta_{i_{\ell}}, \alpha_{i_{\ell}}, \alpha_{i_{\iota}+1}\right)\right)-1\right] .
\end{aligned}
$$

Recalling that $\left|e^{z}-1\right| \leqq 2|z|$ for $z \in \mathbb{C},|z|<\frac{1}{2}$, for $\lambda$ varying in a suitable neighborhood $\omega$ of the origin on $\mathbb{C}^{d}$ and $L$ sufficiently large [cf. Eq. (2.15)]

$$
\begin{aligned}
\left|\zeta_{\lambda}(R)\right| \leqq \sum_{\beta_{\tilde{R}}} \prod_{B_{i} \in \tilde{R}}\left[\frac{\exp \left(J^{\Phi}\left(\beta_{i}, \alpha_{i}, \alpha_{i+1}\right)\right.}{\sum_{\beta_{l}} \exp \left(J^{\Phi}\left(\beta_{i}, \alpha_{i}, \alpha_{i+1}\right)\right.}\right] \\
\cdot \prod_{s=1}^{k} 2\left|W_{C_{s}}^{\Phi+\Psi_{\lambda}}\left(\alpha_{C_{s}}, \beta_{C_{s}}\right)\right| \prod_{\ell=1}^{h} 2\left|J^{\Psi_{\lambda}}\left(\beta_{i}, \alpha_{i}, \alpha_{i+1}\right)\right| .
\end{aligned}
$$

Expressing each $W$ and $J$ in terms of the original occupation numbers $x_{t}$ and performing the products $\prod_{s}$ and $\prod_{\ell}$, we get a sum of terms of the form

$$
\Phi\left(I_{i_{1}} \cup \ldots \cup I_{i_{p_{1}}}\right) \ldots \Phi\left(I_{p_{j}+1} \cup \ldots \cup I_{p_{j+1}}\right) \ldots \Phi\left(I_{i_{h+k-1}+1} \cup \ldots \cup I_{i_{h+k}}\right) \cdot \prod_{r=1}^{h+k} \prod_{t \in I_{r}} x_{t} .
$$

Noticing that from the definition of a polymer, it follows that each term of the sum contains at least one factor $x_{t}$ for each block $B$ belonging to $R$,

$$
\begin{gathered}
\left|\zeta_{\lambda}(R)\right| \leqq Q^{\tilde{R}} \prod_{s=1}^{k} 2\left\|W_{C_{s}}^{\Phi+\Psi} \lambda\right\| \prod_{i=1}^{h} 2\left\|J_{i}^{\Psi} \lambda\right\|, \\
\varrho=\sup _{i} \sup _{I \subset B_{i}} \sup _{\alpha_{i}, \alpha_{i+1}} \sum_{\beta_{i}} \prod_{t \in I} x_{t} \frac{\exp \left(J^{\Phi}\left(\beta_{i}, \alpha_{i}, \alpha_{i+1}\right)\right)}{\sum_{\beta_{i}} \exp \left(J^{\Phi}\left(\beta_{i}, \alpha_{i}, \alpha_{i+1}\right)\right)} \\
\leqq(1+\exp (-\|\Phi\| \mid))^{-1} .
\end{gathered}
$$

The last estimate follows from

$$
\begin{gathered}
\sum_{x_{B^{\prime} \backslash t}} \exp \left(\sum_{Y \subset \hat{B}_{l}}[\Phi(Y \cup t)+\Phi(Y)] \prod_{r \in Y} x_{r}\right) \\
\sum_{x_{\hat{B}_{i} \backslash t}} \exp \left(\sum_{Y \subset \hat{B}_{\imath}}[\Phi(Y \cup t)+\Phi(Y)] \prod_{r \in Y} x_{r}\right)+\sum_{x_{B_{\imath} \backslash t}} \exp \left(\sum_{Y \subset \hat{B}_{t}} \Phi(Y) \prod_{r \in Y} x_{r}\right) \\
\leqq\left[1+\inf _{x_{B_{l}} \backslash t} \exp \left(\sum_{Y \subset \hat{B}_{l}} \Phi(Y \cup t) \sum_{r \subset Y} x_{r}\right)\right]^{-1} \leqq(1+\exp (-\|\Phi\|)]^{-1}
\end{gathered}
$$


where

$$
\hat{B}_{i}=B_{i} \cup A_{i} \cup A_{i+1} .
$$

An analogous argument works for the polymers $S$ introduced in the proof of the theorem. We have:

$$
\Theta(S)=\sum_{\alpha \tilde{s}} \prod_{A_{l} \in \tilde{S}} \frac{\exp \left[\bar{a}^{\Phi}\left(\alpha_{i}\right)\right]}{\sum_{\alpha_{1}} \exp \left[\bar{a}^{\Phi}\left(\alpha_{i}\right)\right]} \prod_{D \subset S} \exp \left[V_{D}\left(\alpha_{D}\right)-1\right] .
$$

The difference with the case previously discussed lies in the fact that the interactions contributing to the $V$ 's can be of different form. For instance, we have now terms like $\varphi_{T}\left(R_{1} \ldots R_{n}\right) \prod_{i=1}^{n} \zeta\left(R_{i}\right)$ where the dependence on the configurations can be [cf. (A2)]:

i) via the terms $W^{\Phi+\Psi_{\lambda}}$ and $J^{\Psi_{\lambda}}$, that is of the form $\sum \Phi(X) \prod_{t \in X} x_{t}$ as before;

ii) or via the factors $\exp \left(J^{\Phi}\left(\beta_{i}, \alpha_{i}, \alpha_{i+1}\right)\right) / \sum_{\beta_{i}} \exp \left(J^{\Phi}\left(\beta_{i}, \alpha_{i}, \alpha_{i+1}\right)\right)$, where our previous arguments do not apply.

Given a block $A$ let us classify the interactions involving this block in three classes:

a) interactions where the dependence on the configuration $x_{A_{i}}$ is of the form $\sum_{X} \Phi(X) \prod_{t \in X} x_{t}$

b) the two body terms of the form

$$
U\left(\alpha_{i} \alpha_{i+1}\right)=\log \frac{Z_{B_{\imath}}^{\alpha_{\imath}, \alpha_{i+1}}(\Phi) \cdot Z_{B_{i}}^{0,0}(\Phi)}{Z_{B_{\imath}}^{\alpha_{i}, 0}(\Phi) \cdot Z_{B_{\imath}}^{0, \alpha_{\imath}}(\Phi)}
$$

c) terms coming from

$$
\varphi_{T}\left(R_{1} \ldots R_{n}\right) \prod_{i=1}^{n} \zeta\left(R_{i}\right)
$$

where the dependence from $x_{A_{i}}$ is only via the factor

$$
\exp \left(J^{\Phi}\left(\beta_{i}, \alpha_{i}, \alpha_{i+1}\right)\right) / \sum_{\beta_{i}} \exp \left(J^{\Phi}\left(\beta_{i}, \alpha_{i}, \alpha_{i+1}\right)\right) .
$$

For the interactions of the class b) we can always put

$$
U\left(\alpha_{i}, \alpha_{i+1}\right)=\varrho \bar{U}\left(\alpha_{i}, \alpha_{i+1}\right) \text {, where } \bar{U}\left(\alpha_{i}, \alpha_{i+1}\right)=U\left(\alpha_{i}, \alpha_{i+1}\right) / \varrho
$$

can be made arbitrarily small for $M$ sufficiently large (cf. Lemma 3). For the contributions of the class $\mathrm{c}$ ), considering that in a given polymer $R$ there are at most $2|\tilde{R}| A$-blocks involved via the $e^{J} / \sum e^{J}$ factors we can write:

$$
\left|\varphi_{T}\left(R_{1} \ldots R_{n}\right)\right| \prod_{i=1}^{n}\left|\zeta\left(R_{i}\right)\right| \leqq(\sqrt{\varrho})^{F / 2}\left|\varphi_{T}\left(R_{1} \ldots R_{n}\right)\right| \prod_{i=1}^{n}\left|\zeta\left(R_{i}\right)\right|
$$

where $F$ is the number of the $A$-blocks nearest neighbor of the $B$-blocks in the chain of polymers $R_{1}, \ldots, R_{n}$ and

$$
\tilde{\zeta}(R)=\left(\frac{1}{\sqrt{\varrho}}\right)^{|\tilde{R}|}|\zeta(R)|
$$


Now writing down the analog of Eq. (A3), we recover the $\varrho$ factors coming from the interactions of the class a) in the usual way, meanwhile the blocks associated only to interactions of the class b) or c) carry their own damping factor $\sqrt{\varrho}$ and $\varrho^{1 / 4}$ respectively. So we get

$$
\Theta(S) \leqq\left(\varrho^{1 / 4}\right)^{|\tilde{S}|} \prod_{D \subset S} \varphi_{D}
$$

where for

$$
D=A_{i}, \varphi_{D}=\sup _{i}\left\|a_{i}^{\Psi_{\lambda}}\right\| \frac{2}{\sqrt{\varrho}}
$$

for

$$
D=A_{i}, A_{i+1}, \varphi_{D}=\frac{2}{\varrho}\left\|V_{A_{i} A_{i+1}}\right\| .
$$

In all the other cases

$$
\varphi_{D}=\left\|W_{D}^{\Phi+\Psi_{\lambda}}\right\|+\left\|\hat{W}_{D}^{\Phi+\Psi_{\lambda}}\right\|
$$

where $\hat{W}$ is the analog of $\tilde{W}\left[\mathrm{cf} .\left(2.19^{\prime \prime}\right)\right]$ where we consider $\tilde{\zeta}(R)$ instead of $\zeta(R)$.

\section{Appendix B}

In this appendix we want to prove Eq. (2.8). For the definitions of the quantities that will appear in the sequel we refer to Sect. 2 . We assume that

with

$$
|\zeta(R)| \leqq \sigma^{|\tilde{R}|} \prod_{C \in R} \varphi_{\mathrm{C}}
$$

$$
0<\sigma<1, \quad \sup _{a \in \tilde{G}} \sum_{C \ni a} \varphi_{C} \leqq K .
$$

We will need some more restriction on $\sigma, K$ [see (2.7), (2.8)].

We want to evaluate

$$
\sum_{R: \tilde{\tilde{R}} \ni a}|\zeta(R)| .
$$

For a given polymer $C_{1}, C_{2}, \ldots, C_{k}$ such that $\tilde{R} \ni a$, call $C_{0}$ an arbitrarily chosen bond $\mathbb{C}$ passing through $a$ (i.e. such that $\tilde{C}_{0} \ni a$ ).

Now we will classify the bonds of a generic polymer containing $C_{0}$ in a "hierarchical" way. Call $C_{1}^{1}, C_{2}^{1}, \ldots, C_{k_{1}}^{1}$ the set of all the bonds in $R$ such that

$$
\forall i=1,2, \ldots, k_{1}, \tilde{C}_{i}^{1} \cap \tilde{C}_{0} \neq \emptyset .
$$

We will refer to $\left\{C_{i}^{1}, i=1,2, \ldots, k_{1}\right\}$ as the "first generation". Notice that the first generation can be empty iff $R$ reduces to $C_{0}$.

We define the "second generation" (if any) $C_{1}^{2}, C_{2}^{2}, \ldots, C_{k_{2}}^{2}$ as the set of all bonds in $R$ such that

$$
\tilde{C}_{j}^{2} \cap\left(\bigcup_{i=1}^{k_{1}} \tilde{C}_{i}^{1}\right) \neq \emptyset \quad j=1,2, \ldots, k_{2},
$$


i.e. the set of all bonds passing through the "first generation". It is implicit in the definition that

$$
\tilde{C}_{j}^{2} \cap \tilde{C}_{0}=\emptyset \quad j=1,2, \ldots, k_{2} .
$$

We can define recursively the $h^{\text {th }}$ generation $C_{1}^{h}, C_{2}^{h}, \ldots C_{k_{h}}^{h}$ as the set of all bonds in $R$ such that

$$
\tilde{C}_{j}^{h} \cap\left(\bigcup_{i=1}^{k_{h}-1} \tilde{C}_{i}^{h-1}\right) \neq \emptyset
$$

with

$$
\tilde{C}_{j}^{h} \cap\left(\bigcup_{\ell=1}^{h-2} \bigcup_{i=1}^{k_{\ell}} \tilde{C}_{i}^{\ell}\right)=\emptyset .
$$

For any given polymer $R$ containing $C_{0}$, a finite integer $t \geqq 0$, will be uniquely determined expressing the number of generations present in $R$.

We can then write:

$$
\begin{aligned}
& \sum_{R: \tilde{\hat{R}} \ni a}|\zeta(R)| \leqq \sum_{C_{0} \ni a}\left|\zeta\left(C_{0}\right)\right| \\
& \quad+\sum_{C_{0} \ni 0} \sum_{t=1}^{\infty} \sum_{k_{1}=1}^{\infty} \sum_{C_{1}^{1} \ldots C_{k_{1}}^{1}} \ldots \sum_{k_{t}=1}^{\infty} \sum_{C_{1}^{1} \ldots C_{h^{\prime}}}\left|\zeta\left(R^{*}\right)\right|,
\end{aligned}
$$

where the sum in the right hand side of (B3) are performed with the constraints specified by $\left(\mathrm{B} 1^{\prime}\right),\left(\mathrm{B} 2^{\prime}\right)$ and $R^{*}$ is the polymer $C_{0}, C_{1}^{1}, \ldots, C_{k_{1}}^{1}, \ldots, C_{1}^{t}, \ldots, C_{k_{t}}^{t}$.

Now for a given $t$ let us start summing over the $t^{\text {th }}$ generation supposing all the other bonds belonging to the previous generations to be fixed. It is easy to convince oneself that

$$
\begin{aligned}
& \sum_{k_{t}=1}^{\infty} \sum_{C_{1}^{t} \ldots C_{k_{t}}^{t}}\left|\zeta\left(R^{*}\right)\right| \\
& \leqq \sigma^{\left|\Delta_{1}\right|+\ldots+\left|\Delta_{t-1}\right|} \prod_{j=1}^{t-1} \prod_{\ell=1}^{k_{J}} \varphi_{C_{\ell}^{j}} \prod_{B \in \Delta_{t-1}}\left[\sum_{n=0}^{\infty}\left(\sum_{C \ni B} \varphi_{C}\right)^{n} \frac{1}{n !}-1\right] \\
& \leqq \sigma^{\left|\Delta_{1}\right|+\ldots+\left|\Delta_{t-1}\right|} \prod_{j=1}^{t-1} \prod_{\ell=1}^{k_{j}} \varphi_{C_{\ell}}\left[\exp K\left|\Delta_{t-1}\right|-1\right]
\end{aligned}
$$

where

$$
\Delta_{s}=\bigcup_{h=1}^{k_{s}} \tilde{C}_{h}^{s} \backslash \bigcup_{h=1}^{k_{s}-1} \tilde{C}_{h}^{s-1}, \quad s=1, \ldots, t
$$

Now summing over the $t-1^{\text {th }}$ generation we obtain

$$
\begin{aligned}
& \sum_{k_{t}-1=1}^{\infty} \sum_{C_{1}^{t-1}} \sum_{C_{t_{1}-1}^{t-1}} \sum_{k_{t}=1}^{\infty} \sum_{C_{1}^{t} \ldots C_{k_{t}}^{t}}\left|\zeta\left(R^{*}\right)\right| \\
& \leqq \sigma^{\left|\Delta_{1}\right|+\ldots+\left|\Delta_{t}-2\right|} \prod_{j=1}^{t-2} \prod_{\ell=1}^{k_{j}} \varphi_{C_{t}^{j}} \sum_{N_{t-1}=1}^{\infty}\left(\exp \left(K N_{t-1}\right)-1\right) \\
& \cdot \sigma^{N_{t-1}} \sum_{k_{t-1}=1}^{\infty} \sum_{C_{1}^{t-1} \ldots C_{k_{t-1}}^{-1}}^{*} \prod_{\ell=1}^{k_{t-1}} \varphi_{C_{\ell}^{t-1}},
\end{aligned}
$$


where the $*$ sum is over all the sets of bonds $C_{1}^{t-1} \ldots C_{k_{t-1}}^{t-1}$, such that $\left|\Delta_{t-1}\right|=N_{t-1}$ and, of course, the condition (B1') is satisfied. Now relaxing the condition we can proceed exactly as in (B4) and obtain:

$$
\begin{aligned}
& \sum_{k_{t-1}=1}^{\infty} \sum_{C_{t}^{t-1}} \sum_{C_{t_{t-1}^{\prime-1}}} \sum_{k_{t}=1}^{\infty} \sum_{C_{1}^{t} \ldots C_{t_{t}}^{t}}\left|\zeta\left(R^{*}\right)\right| \\
& \leqq \sigma^{\left|\Delta_{1}\right|+\ldots+\left|\Delta_{t-2}\right|} \prod_{j=1}^{t-2} \prod_{\ell=1}^{k_{j}} \varphi_{C_{\ell}}\left(\exp \left(K\left|\Delta_{t-2}\right|\right)-1\right) \sum_{N_{t-1}=1}^{\infty}\left(\exp \left(K N_{t-1}\right)-1\right) \sigma^{N_{t-1}}
\end{aligned}
$$

Iterating this procedure we finally get:

$$
\begin{aligned}
\sum_{R: \tilde{R} \ni a}|\zeta(R)| \leqq & \sum_{C_{0} \ni a} \sigma \varphi_{C_{0}}+\sum_{C_{0} \ni a} \varphi_{C_{0}} \sigma^{\left|C_{0}\right|}\left(\exp \left(K\left|C_{0}\right|\right)-1\right) \\
& \cdot \sum_{t=1}^{\infty}\left[(\exp (K n)-1) \sigma^{n}\right]^{t-1} \\
\leqq & K \sigma\left[1+\frac{\exp K-1}{1+\sigma^{2} \exp K-2 \sigma \exp K}\right]
\end{aligned}
$$

if

\section{Appendix C}

$$
\exp K<[\sigma(2-\sigma)]^{-1}
$$

Proof of Lemma 3. In this proof we will use not only the notations but also the equations of Ruelle's paper and denote them by $(\cdot, \cdot R)$. Let

$$
N^{+}=\{i \in \mathbb{Z}: i>0\}, \quad K^{+}=\{0,1\}^{N^{+}},
$$

$C\left(K^{+}\right)=$Banach space of real continuous functions on $K^{+}$. For $x_{N^{+}} \in K^{+}$and

$$
x_{A^{-}} \in\{0,1\}^{L}, A^{-}=\{t \in \mathbb{Z},-L+1 \leqq t \leqq 0\}
$$

call

$$
\begin{gathered}
W\left(x_{A^{-}} \mid x_{N^{+}}\right)=\sum_{\substack{\emptyset \neq X \subset A^{-} \\
\theta \neq Y \subset N^{+}}} \Phi(x \cup Y) \prod_{t \in X \cup Y} x_{t} \\
f_{x_{A^{-}}} \in \mathscr{C}\left(K^{+}\right): f_{x_{A^{-}}}\left(x_{N^{+}}\right)=\exp W\left(x_{A^{-}} \mid x_{N^{+}}\right) .
\end{gathered}
$$

If

$$
x_{N^{+}} \subset K^{+}
$$

define $\left(x_{A^{+}}, x_{N^{+}}\right)=\left(x_{A^{+}}, T^{L} x_{N^{+}}\right)$, where $T$ is the operator that translates the configuration in $K^{+}$by one unit to the right [cf. (1.16R) and (2.1R)] and put

$$
x_{0} \equiv(0, \ldots, 0, \ldots) \in K^{+} \text {. }
$$

It is easy to see that

$$
\begin{aligned}
\log \frac{Z_{B_{l}}^{\alpha_{1}, \alpha_{i+1}}(\Phi) \cdot Z_{B_{l}}^{0,0}(\Phi)}{Z_{B_{i}, 0}^{\alpha_{i}}(\Phi) \cdot Z_{B_{i}}^{0, \alpha_{i+1}}(\Phi)} \\
=\log \frac{\left(\mathscr{L}^{M} f_{\alpha_{l}}\right)\left(\alpha_{i+1}, x_{0}\right) \cdot\left(\mathscr{L}^{M} 1\right)\left(x_{0}\right)}{\left(\mathscr{L}^{M} f_{\alpha_{i}}\right)\left(x_{0}\right) \cdot\left(\mathscr{L}^{M} 1\right)\left(\alpha_{i+1}, x_{0}\right)}
\end{aligned}
$$


where $\mathscr{L}$ is the operator on $\mathscr{C}\left(\mathrm{K}^{+}\right)$defined by $(2.3 \mathrm{R})$, then the thesis follows immediately from Eq. (2.6R).

Acknowledgements. We are very indebted to Giovanni Gallavotti for stimulating our interest in this problem and for many helpful discussions. We wish also to thank Donatella Capocaccia and Giosi Benfatto for many useful remarks and comments.

\section{References}

1. Dobrushin, R.L.: Commun. Math. Phys. 32, 269 (1973)

2. Hoghton, A., Kadanoff, L.P.: In : Renormalization group in critical phenomena and quantum field theory. Proceeding of a Conference 1973. Gunton, J.D., Green, M.S. (eds.) Dept. of Phys., Temple University, Philadelphia

Nelson, D., Fisher, M.: Ann. Phys. 91, 226 (1975)

3. Gallavotti, G., Martin-Löf, A., Miracle-Sole, S. : Lecture Notes in Physics 20, 162 (1971)

4. Gruber, Ch., Kunz, H.: Commun. Math. Phys. 22, 133 (1971)

5. Kunz, H.: Commun. Math. Phys. 59, 53 (1978)

6. Campanino, M., Capocaccia, D., Tirozzi, B.: Commun. Math. Phys. 70, 125 (1979)

7. Ruelle, D.: Commun. Math. Phys. 9, 267 (1968)

8. Gallavotti, G., Miracle-Sole, S.: J. Math. Phys. 11, 147 (1968)

9. Isreal, R.B. : Commun. Math. Phys. 50, 245 (1976)

10. Ruelle, D.: Commun. Math. Phys. 18, 126 (1970)

Ruelle, D.: Commun. Math. Phys. 50, 189 (1976)

Communicated by E. Lieb

Received February 5, 1981 
